Ann. Génét. Sél. anim., I977, 9 (2), I5I-16r.

\title{
Hybridation entre un Mouflon de Corse (Ovis ammon musimon Schreber, 1872) et des brebis de divers génotypes : gènes pour la coloration pigmentaire
}

\author{
J.-J. LAUVERGNE, B. DENIS* et M. THÉRET*
}

Département de Génétique animale,

Centre national de Recherches zootechniques, I.N.R.A., $7835^{\circ}$ Jouy-en-Josas, France

(*) Service de Zootechnie, Ecole Nationale Vétérinaire d'Alfort 9470 I Maisons-Alfort, France.

\section{Résumé}

Un Mouflon de Corse mâle a été croisé avec des brebis dont les différents génotypes colorés, aux loci $A$ (Agouti) et $E$ (Extension) - qui contrôlent fondamentalement la pigmentation du pelage des mammifères en général et du mouton en particulier - étaient connus.

On a conclu tout d'abord que le mâle sauvage portait en $E$ l'allèle non muté $E+$ présent généralement chez le mouton domestique, sauf dans les races portant le noir dominant $\left(E^{d} E^{d}\right.$ ou $\left.E^{a} E^{+}\right)$. L'allèle $E^{+}$permet l'expression normale des gènes en $A$ gouti.

Au locus $A$ gouti, le Mouflon porte l'allèle "sauvage " $A^{+}$. Cet allèle est responsable du patron fauve avec charbonnures et ventre blanc de l'espèce sauvage. On a montré que cet allèle est récessif vis-à-vis de $A$ wh (rouge) et dominant avec expressivité variable vis-à-vis de $a$ (noir) $: 5 A+a$ sur 8 avaient le phénotype sauvage à la naissance (comme $A^{+} A^{+}$) et 3 avaient un phénotype intermédiaire, très semblable à "noir et feu ": dos noir ventre blanc. La question de savoir si l'allèle sauvage $A^{+}$peut être confondu avec un allèle déjà identifié chez plusieurs races de mouton et qui donne le "noir et feu " $\left(A{ }^{w}\right)$ a été posée. Une réponse définitive n'a pas été donnée mais il semblerait que les 2 allèles sont distincts, même si l'expression de l'un peut être imitée par l'autre $\left(A+a\right.$ donnant le " noir et feu ", comme $A$ wa ou $A{ }^{w} A$ w) et même si le changement de structure de la toison, en passant de l'espèce sauvage à l'espèce domestique peut expliquer, au moins partiellement, un changement de l'expressivité du gène.

\section{Introduction}

Chez le mouton domestique les deux loci Agouti $(A)$ et Extension $(E)$ qui contrôlent, comme chez tous les Mammifères, la production et la répartition des pigments mélaniques (SEARLE, I968), ont été raisonnablement bien isolés ces vingt dernières années (RENDEL, I957, AdAlsteinsson, I960, I970, I974, NEL, 
i967, Hoogschagen, I967, Brooker et Dolling, I969, Lauvergne, I975).

Toutefois, devenus des "bêtes à laine " (à toison autant que possible blanche) ou des " bêtes à fourrure " (à toison surtout noire ou grise) les moutons domestiques actuels ont un pelage qui, de par sa structure et sa couleur, s'éloigne fort de celui de l'ancêtre sauvage qui est sans doute le Mouflon (Bunch et al., I976). Ainsi, on ne peut guère connaître la situation des allèles sauvages et leur comportement qu'en croisant des moutons domestiques avec le Mouflon. Par le passé, de tels travaux ont déjà été entrepris mais à une époque où l'on ignorait encore l'existence de $A$ et $E$. Ils méritaient d'être repris, d'où la série de croisements expérimentaux qui sert de support au présent article.

\section{Matériel et méthodes}

\section{A. - L'origine des données}

Les données proviennent d'une expérience entreprise, au départ, pour l'étude de la mue spontanée de la toison. Un Mouflon mâle, originaire de Corse, a été croisé à des brebis Berrichonnes du Cher et Limonsines. Des $\mathrm{F}_{2}$ et des croisements en retour (back-cross) sur le Mouflon et sur les deux races maternelles ont également été faits. Les premiers hybrides sont nés en I973 et les dernières naissances datent de I977.

L'étude a été complétée par le croisement du même Mouflon avec des brebis provenant d'une expérience conduite à Marmilhat (cf. LAUvERGNE, r975) et dont les formules génotypiques pour la couleur de la robe étaient connues, ainsi qu'avec des brebis Solognotes noires apparues dans des troupeaux de couleur rouge, habituelle en cette race. Les naissances ont été obtenues en 1977 .

L'ensemble des travaux expérimentaux a été conduit au Service de Zootechnie de l'École Vétérinaire d'Alfort.

\section{B. - Les phénotypes observés}

\section{Description}

- Le patron "sauvage " du Mouflon de Corse (Ovis ammon musimon, ScHreBER, I782) est décrit par PFEFFER (I967) comme suit : " le pelage est caractérisé en hiver par une teinte générale brune plus ou moins foncée, la présence chez le mâle adulte d'une tache dorsale ou " selle » blanche... Le ventre, la région caudale, l'intérieur et l'extrémité des membres, la mâchoire inférieure et la face sont blancs. Les femelles sont, dans l'ensemble, plus claires que les mâles ».

En ouvrant le pelage on observe l'existence, dans certaines régions, de poils zonés noirs à la base fauves au sommet. L'extension vers le sommet du poil de la zone noire est responsable des charbonnures que l'on observe le cas échéant sur la tête, le devant des membres et, souvent, la ligne du dessus (fig. I, $a$ ).

- Le patron " noir et feu ". C'est le terme introduit par l'un de nous (LAUVERGNe, I970, LAUVERGNe et AdALsteinsson, I976) pour désigner chez le mouton une variation communément dite "black and tan " chez les Mammifères : dos noir, ventre et intérieur des membres rouges ou blancs (SEARLE, I968). Roberts 
et JENKIn (I926) le nomment " reversed badger face " terme auquel BERGE (I958) préfère "black mouflon ", de même que AdALsTEInsson (I970). Au cours de nos expériences un certain nombres de variations de ce phénotype, qui est présenté dans la figure $\mathrm{I}, b$, ont été observées.

- Patron " rouge ", souvent dilué est assorti d'une panachure blanche assez étendue (fig. I, $c$ ).

- Patron noir (fig. I, $d$ ).

- Patron blanc.
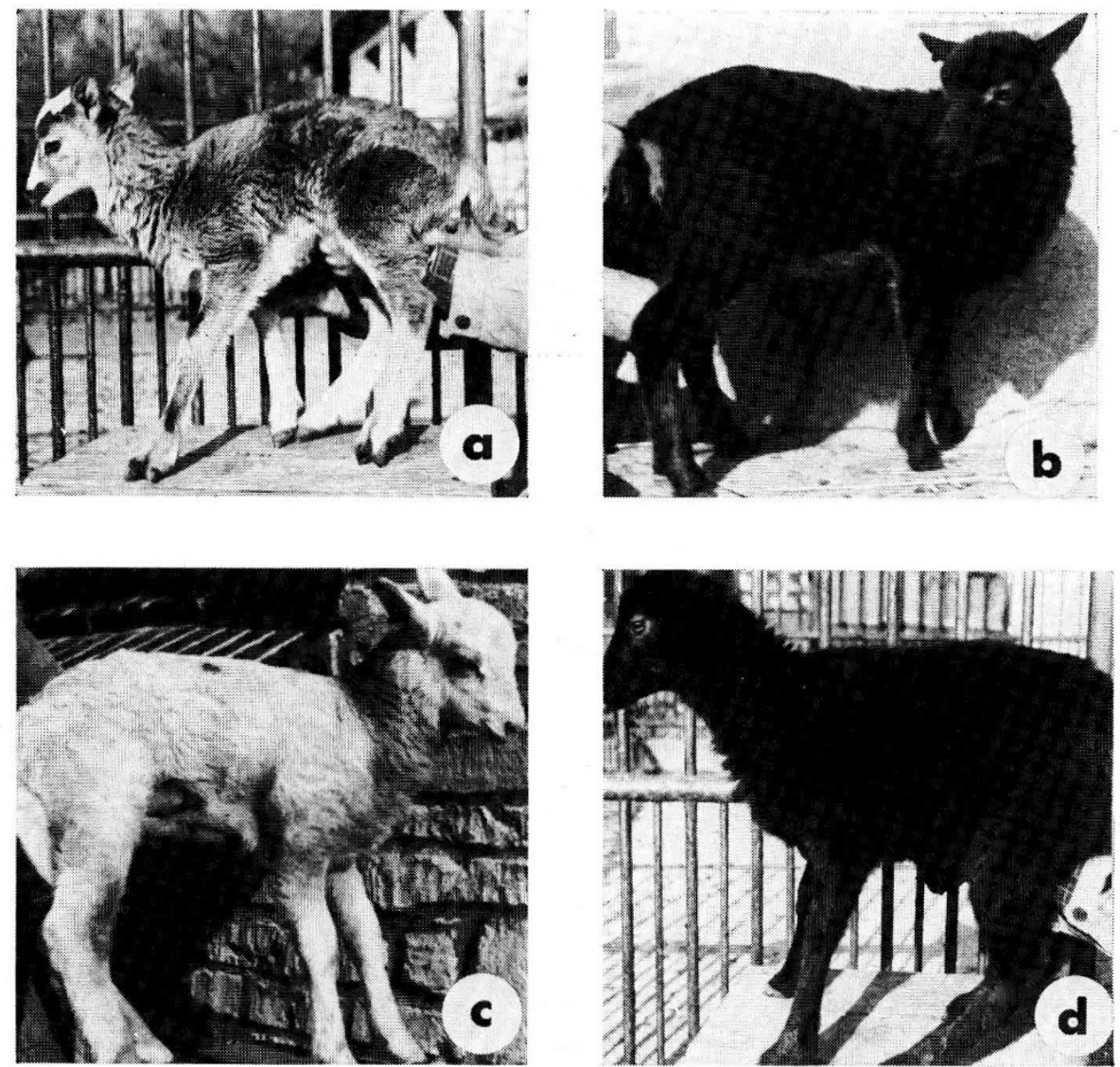

FIG. I. - Les phénotypes pigmentaires vencontrés au cours des expériences de croisement. a) "sauvage ", b) " noir et feu ", c) rouge (pie), d) noir.

Pigmentary phenotypes in the crossing experiment.

a) " wild ", b) " black and tan ", c) red (piebald), d) black.

2. Variation avec l'âge et la structure de la toison

Une canitie plus ou moins précoce se manifeste, surtout chez les patrons rouge ou pie rouge.

Dans certains cas on a noté, au contraire, un renforcement de la couleur avec 
l'âge. C'est ainsi que deux animaux nés « sauvages » sont par la suite devenus " noir et feu ".

Il est probable que certaines variations de colorations sont dues à la structure même de la toison, certain type de mélanine diffusant plus ou moins selon le type de production pileuse. Il avait déjà été remarqué (ADALsTEInsson, I970) que la mélanine rouge diffusait d'autant plus mal que le diamètre du brin était plus fin. Ce pourrait être le contraire pour la mélanine noire, ce qui rendrait compte $\mathrm{du}$ noircissement de certains patrons sauvages, avec la pousse de la laine.

\section{Notation des couleurs}

Pour l'analyse des ségrégations on s'est basé, en premier lieu, sur des examens à la naissance mais, les animaux ayant été pour la plupart conservés, on a pu enregistrer et tenir compte de certains phénomènes, en particulier l'assombrissement du "sauvage " en " noir et feu ".

\section{C. - Formules mendéliennes des géniteurs de départ et ségrégations attendues}

\section{Les brebis}

L'étude faite à Marmilhat (LAUVERGNe, I975) donne la formule colorée des femelles utilisées dans la présente étude pour 3 loci : Extension $(E)$, Agouti $(A)$ et Panachure irrégulière $(S)$.

L'article étant consacré à la seule coloration pigmentaire nous ne retiendrons que les loci $A$ et $E$, en rappelant seulement que le blanc est dû à l'interaction de $S^{b}$ avec $A^{w h}$, donc que tous les animaux présentant cette dépigmentation totale sont soit $A^{w h} A^{w h}$, soit $A^{w h} a$.

Ainsi ont été identifiés les allèles suivants :

- Au locus $E$ d'Extension: l'allèle $E^{d}$, dominant pour le noir uniforme et épistatique sur le locus $A$ gouti et l'allèle $E^{+}$qui permet la libre expression des génotypes $A$ gouti.

- Au locus $A$ (Agouti) : l'allèle $A{ }^{w h}$ (rouge) donnant, nous venons de le voir, en interaction avec $S^{b}$, une panachure irrégulière ou même le blanc total et l'allèle $a$ pour le noir, récessif par rapport à $A^{w h}$.

De cette manière, les brebis Berrichonnes $d u$ Cher et Limousines blanches sont $A^{w h} A^{w h} E^{+} E^{+}$ou $A^{w h} a E^{+} E^{+}$(il y en a en effet toujours quelques noirs qui ségrègent dans ces races). Les brebis Solognotes noires ségrégeant dans une race rouge (A ${ }^{w h} A{ }^{w h} E^{+} E^{+}$ou $A^{w h} a E^{+} E^{+}$) sont $a a E^{+} E^{+}$. La race Bizet, noire, ayant pour formule $A^{\text {wh }} A^{\text {wh }} E^{d} E^{d}$ (LAUvergne. I975), les Bizet $\times$ Solognot noires sont $A^{\text {wh }} A^{\text {wh }}$ $E^{d} E^{+}$.

Quant aux métisses Finnois $\times$ Bizet, elles peuvent être soit $A^{w h} A^{w} E^{d} E^{+}$, soit $A^{w h} a E^{a} E^{+}$car le bélier Finnois était $A^{w} a E^{+} E^{+}$. La formule de deux d'entre clles a pu être établie en examinant leur descendance (I027 et II29, qui se sont avérées être $\left.A^{w h} a E^{d} E^{+}\right)$. Celle de 2038 restant encore imprécise, on ne pourra utiliser ses produits que pour tester la ségrégation $E^{d} / E^{+}$. 


\section{Le Mouflon de Corse}

Par définition, l'espèce sauvage porte aux deux loci considérés les allèles sauvages : en $A$ gouti l'allèle responsable du dessin fauve avec charbonnures et ventre clair $A^{+}$, au locus d'Extension l'allèle $E^{+}$qui permet l'expression normale des génotypes en $A$ gouti.

\section{Les ségrégations attendues}

\section{- ordre de dominance probable.}

D'après les observations des anciens auteurs qui ont croisé des races blanches $\left(A^{w h} A^{w h}\right)$ à des moutons sauvages, l'allèle $A^{w h}$ semble dominer $A^{+}$: les $\mathrm{F}_{\mathbf{1}}$ sont en général pie rouge (BUTARIN, I938, WARWIck et al., I957, Young et MANville, I960, NEL, I962). Quant au génotype $A^{+} a$, il a pu être celui de certains croisés rencontrés par KoCH (I937) et WARwICK et al. (I957), il correspondrait alors aux patrons que nous avons nommé "sauvage " et " noir de feu ".

- les ségrégations en $F_{1}$.

Il est plus clair de donner un tableau des ségrégations attendues correspondant aux différents génotypes de brebis (tableau I).

TABLEAU I

Génotypes et phénotypes pigmentés attendus dans les croisements du mâle Mouflon de Corse avec des brebis de différents génotypes

Waited pigmentary phenotypes and genotypes in crossing the Corsican Mouflon ram with ewes of different genotypes

\begin{tabular}{|c|c|c|c|c|c|}
\hline \multicolumn{2}{|c|}{ Femelles } & \multicolumn{4}{|c|}{ Produits issus du Mouflon } \\
\hline Race ou type & Formule & Formule & $\begin{array}{c}\text { Fréquence } \\
\text { p. } 100\end{array}$ & Génotypes & $\begin{array}{c}\text { Phéno- } \\
\text { types }\end{array}$ \\
\hline $\begin{array}{l}\text { Berrichon du Cher } \\
\text { ou Limousin } \\
\text { Solognot noir }\end{array}$ & $\begin{array}{c}\mathrm{E}+\mathrm{E}+\mathrm{A} w h \mathrm{~A} w h \\
\mathrm{E}+\mathrm{E}+\mathrm{A} w h a \\
\mathrm{E}+\mathrm{E}+a a\end{array}$ & $\begin{array}{l}\mathrm{E}+\mathrm{E}+\mathrm{A}+\mathrm{A} w h \\
\mathrm{E}+\mathrm{E}+\mathrm{A}+a \\
\mathrm{E}+\mathrm{E}+\mathrm{A}+a\end{array}$ & $\begin{array}{l}>90 \\
\text { faible } \\
\text { Ioo }\end{array}$ & $\begin{array}{l}\mathrm{A}+\mathrm{A} w h \\
\mathrm{~A}+a \\
\mathrm{~A}+a\end{array}$ & $\begin{array}{c}\text { rouge } \\
? \\
?\end{array}$ \\
\hline $\begin{array}{l}\text { Solognot } \\
\times \text { Bizet } \\
\text { Finnois } \\
\times \text { Bizet }\end{array}$ & $\begin{array}{l}\mathrm{E} d \mathrm{E}+\mathrm{A} w h{ }^{n} w h \\
\mathrm{E} d \mathrm{E}+\mathrm{A} w h a\left({ }^{2}\right)\end{array}$ & $\begin{array}{l}\mathrm{E} d \mathrm{E}+\mathrm{A} w h \mathrm{~A} w h \\
\mathrm{E}+\mathrm{E}+\mathrm{A}+\mathrm{A} w h \\
\mathrm{E} d \mathrm{E}+\mathrm{A}+a \\
\mathrm{E} d \mathrm{E}+\mathrm{A}+\mathrm{A} w h \\
\mathrm{E}+\mathrm{E}+\mathrm{A}+a \\
\mathrm{E}+\mathrm{E}+\mathrm{A}+\mathrm{A} w h\end{array}$ & $\begin{array}{l}50 \\
50 \\
25 \\
25 \\
25 \\
25\end{array}$ & $\begin{array}{l}\mathrm{E} d_{-} \\
\mathrm{A}+\mathrm{A} w h(1) \\
\mathrm{E} d_{-} \\
\mathrm{E} d_{-} \\
\mathrm{A}+a(1) \\
\mathrm{A}+\mathrm{A} w h(1)\end{array}$ & $\begin{array}{c}\text { noir } \\
\text { rouge } \\
\text { noir } \\
\text { noir } \\
? \\
\text { rouge }\end{array}$ \\
\hline
\end{tabular}

(I) En I la formule est $E^{+} E^{+}$qui permet l'expression des allèles en $A$ gouti.

(2) Formule déterminée par examen de la descendance des deux brebis utilisées pour l'étude des ségrégations en $A$. 


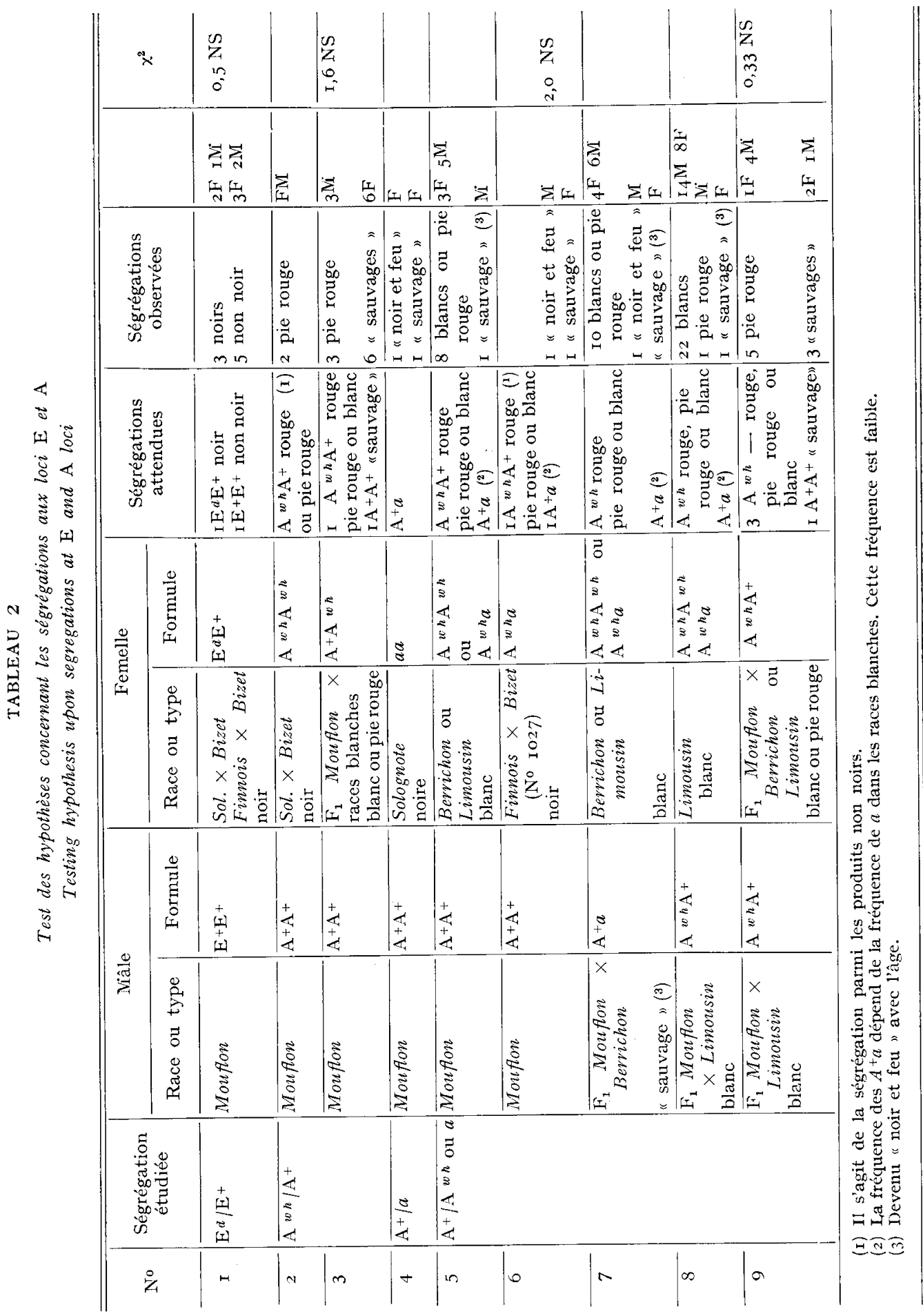


- les ségrégations en back cross et $\mathrm{F}_{2}$.

Les back cross et $\mathrm{F}_{2}$ n'ont été faits qu'entre Mouflon et brebis blanches ils ne permettent que l'étude des ségrégations en $A$ avec principalement $A^{w h}$ et $A^{+}$et, éventuellement, $a$, si les produits de $\mathrm{F}_{1}$ ont révélé l'existence, en races blanches, de quelques hétérozygotes.

\section{Résultats}

Les résultats des croisements comparés aux ségrégations attendues dans les hypothèses que nous venons d'établir sont donnés dans le tableau 2 .

\section{Discussion}

\section{A. - Vérification des hypothèses}

L'examen du tableau 2 (croisement no I) laisse à penser que le Mouflon porte bien l'allèle $E^{+}$en $E$ et que l'allèle $E^{d}$ (noir dominant) en provenance du Bizet a bien, sur $A^{+}$, le même effet épistatique que sur les autres allèles en $A$ gouti, $\left(E^{a}-A^{+} A^{+}\right.$est noir).

L'examen des croisements 2 à 9 (tableau 2) montre bien que l'on peut parfaifaitement admettre l'hypothèse selon laquelle le patron sauvage du Mouflon est dû à un allèle à la série $A$ gouti (déjà marquée par $A^{w h}$ et $a$ ) avec dominance de $A^{w h}$ sur $A^{+}$.

Quant au comportement de $A^{+}$vis-à-vis de $a$, on constate qu'il est dominant avec une expressivité variable. Cela est déjà révélé par le croisement 4 Mouflon $\left(A^{+} A^{+}\right)$par Solognote noire $(a a)$ qui donne un "sauvage " qui rappelle le Mouflon $\left(A^{+}\right)$et un " noir et feu " phénotype intermédiaire entre le "sauvage ", dorst il a le ventre blanc, et le tout noir, dont la couleur envahit le dos à la place du fauve avec charbonnure.

Au total, parmi 8 animaux de génotype $A+a$, on relève 5 " sauvages " et 3 " noir et feu ». Il s'agit, rappelons-le de classifications faites à la naissance et l'on doit ajouter que, par la suite, deux des 5 "sauvages " sont devenus " noir et feu " avec l'âge.

Adalsteinsson (I970) a analysé en détail les rapports de dominance entre 6 allèles de la série $A$ gouti du mouton et montré les ressemblances avec la Souris domestique et, plus généralement, les autres Mammifères. D'une manière générale on observe la dominance de l'inhibition de la production de l'eumélanine noire sur la production d'eumélanine. Souvent existent des allèles qui cumulent les effets de deux allèles différents. Par ailleurs, les hétérozygotes peuvent également cumuler les effets des deux allèles qu'ils portent comme le "black badger face "/ " mouflon " qui combine l'inhibition d'eumélanine de " noir et feu " (sur le ventre) à celle de " blaireau " sur le dos pour ne laisser subsister qu'une raie noire le long du flanc.

Le comportement de $A^{+} a$, qui donne un intermédiaire entre les génotypes des deux allèles, s'apparente avec les observations de l'auteur islandais renouvelées 
depuis, sur le troupeau Corse cette fois (LAuvergne et Adalsteinsson, I976). Simplement on note que l'inhibition de production d'eumélanine sur le dos n'est que partiellement réalisée chez certains animaux, transitoire chez d'autres et peut être alors due à la diffusion différentielle des divers types de mélanine selon le diamètre du brin de laine ou de poil.

Les observations de Koch (I937) correspondent tout à fait aux nôtres. L'animal à phénotype "sauvage " apparu dans un troupeau de Bergschaf en Bavière était probablemens $A^{+} a$. Il présentait à la naissance un patron "sauvage " qui s'est assombri par la suise pour donner une sorte de " noir et feu ». Celles de WARwick et al. (I957) ne sont malheureusement pas très précises, mais il n'est pas impossible que ces auteurs, en croisant les descendants d'un Mouflon avec des brebis portant le noir récessif aient observés des "sauvages " correspondant à ce qu'ils nomment " blackish ) déjà AdALSTEInsson (I974) a fait la remarque qu'il pourrait s'agir de rouge ou de pie rouge et même des " noir et feu " nommés " pie noir ". Mais ce n'est qu'une impression qui se base sur l'examen de deux photos données dans l'article. Roberts (I93I) quant à lui n'a observé que des noirs uniforme (issus d'un noir dominant) et des " noir et feu " mais ne sait pas exactement la formule en $A$ gouti des brebis de départ et la discussion sera reprise plus loin.

\section{B. - Un seul et même facteur pour les patrons " sauvage " et "noir et feu " ou bien deux allèles}

S'appuyant sur le fait qu'en $\mathrm{F}_{\mathbf{1}}$ et $\mathrm{F}_{2}$ Mouflon $\times$ Welsh Mountains il observait outre des noirs et des blancs, des "reversed badger face " (" noir et feu ") RoBERTs (I93I), pensait que le Mouflon portait un facteur responsable de ce dessin. Les interprétations de WARWICK et al. (I957) dont nous venons de faire mention concernant des croisés $F_{1}$ et des back cross avec des brebis de races blanches dont certaines portaient le noir récessif sont loin d'être claires, avec une possible confusion dans l'identification des phénotypes.

En fait, comme nous l'avions déjà dit plus haut, ces auteurs qui, par le passé, sont les seuls à avoir pu disposer de données $\mathrm{F}_{1}$ et de back cross ou $\mathrm{F}_{2}$ entre Mouflon et Mouton domestique, ignoraient encore le mécanisme des séries $A$ et $E$ pour expliquer l'essentiel de la pigmentation du Mouton.

Retrouvant en race Spaelsau (le vieux mouton norvégien) le patron "noir et feu ", BERGE (I958), s'inspirait des observations et des conclusions de ROBERTS (I93I) et considérait ce patron comme celui de l'espèce sauvage, le renommant " mouflon ", sans pour autant placer le facteur responsable au locus A gouti, locus que cet auteur, comme les auteurs précédemment cités, ne cerne et cernera (BERGE, I964, I974) que très sommairement, ne faisant guère appel à la notion d'homologie entre loci de coloration des mammifères.

Isolant, pour la première fois sans ambiguité, la série Agouti, AdalsteinsSON (I960) montre, quant à lui, que le facteur responsable du " reversed badger face " (notre " noir et feu ") appartient à cette série et il lui attribue le symbole $A_{4}$. Plus tard, se ralliant aux vues de BERGE, cet auteur en viendra à considérer cet allèle comme l'allèle sauvage, le renommant " mouflon " ou " mouflon noir ", Adalsteinsson (I970). Sans aller toutefois jusqu'à lui donner le symbole $\mathrm{A}^{+}$, SEARLE (I968) lui aussi n'est pas loin de considérer cet allèle comme l'allèle sauvage. Le terme qu'il propose $(A w)$ sera par la suite repris par AdALsternsson (I974) en remplacement de $A$.

L'assimilation par BERGE du patron "noir et feu » au patron "sauvage " de 
l'espèce que Adalsteinsson a traduite en terme factoriel peut paraître à première vue exagérée car, dans de nombreuses espèces de Mammifères on a pu démontrer que ces deux patrons étaient régis par deux allèles différents, généralement nommés $A^{+}$et $A^{t}$ (SEARLE, I968).

Cependant, avec le genre Ovis, pour l'expressivité d'un gène qui passe de l'espèce sauvage à l'espèce domestique, il faut tenir compte de la différence considérable dans la structure du pelage entre l'espèce sauvage et l'espèce domestique qui fait que la coloration peut être considérablement modifiée, comme nous l'avons évoqué ci-avant. Supposant, à la limite, que, chez certains Mouflons il y ait un sous-poil noir totalement masqué par les poils de garde plus ou moins fauves, l'animal paraîtra bien fauve à ventre blanc mais, si l'on transpose ce patron sur une toison uniquement formée de laine (qui correspond au sous-poil) on risque d'avoir un animal " noir et feu ». Toutefois cette situation limite ne semble pas être atteinte car Adalsteinsson (I970) fait état d'une grande variabilité du patron que cet auteur nomme " mouflon ": depuis notre " noir et feu " jusqu'à notre " sauvage ". Est-ce à dire pour autant que l'allèle $A$ w de l'auteur islandais correspond à notre allèle $A^{+}$, les $A{ }^{w} A$ w étant "sauvages " les $A$ wa étant " noir et feu " ou intermédiaires? Cela n'est pas si sur car il est des lignées comme le Soay du Muséum de Paris qui semblent homozygotes et qui présentent le seul patron " noir et feu ". Par ailleurs la race Islandaise étant multisegrégante pour la couleur l'isolément des allèles n'est pas toujours aisé et un regroupement phénotypique un peu étendu peut faire que deux allèles restent confondus.

Les observations ce RoBERTs (I93I) s'expliquant de la même manière - par cette interaction de $A^{+}$avec $a$ qui donne un phénotype rappelant celui dérivant d'un autre allèle - on s'aperçoit que la vision des précédents auteurs n'était pas dénuée de tout fondement mais qu'elle ne s'oppose pas fondamentalement à une interprétation triallélique $A^{+}, A^{w}, a ;(A w$ étant le gène pour le " noir et feu ") plus conforme avec les règles de l'homologie pour la série $A$ gouti.

D'après nos observations à Marmilhat (LAUverGne, I975) ce possible allèle " noir et feu " $A^{w}$ serait dominant sur a. Il faudrait maintenant le mettre en présence de $A^{+}$en croisant par exemple le Mouflon avec une race homozygote pour " noir et feu » par exemple la lignée Soay du Muséum d' Histoire naturelle, à laquelle nous avons déjà fait allusion.

\section{Conclusion}

Même si on ne peut définitivement statuer il est fort probable que l'allèle sauvage $A^{+}$porté par le Mouflon au locus $A$ gouti est distinct d'un allèle muté induisant le phénotype "noir et feu " chez le mouton domestique. Ainsi, $A^{+}$viendrait s'ajouter à la liste déjà longue des allèles en A gouti (7 termes à l'issue de l'étude de Lauvergne et Adalsteinsson, I976, en Corse). Cette situation de polyallélisme au locus $A$ gouti est bien en accord avec la situation généralement observée chez les mammifères domestiqués où le locus Agouti est celui des loci de coloration dont les mutants sont les plus nombreux à s'être conservés, sinon à être apparus. (SEARLE, I968), le record étant tenu par la Souris avec I3 allèles distincts (SEARLE, I976). 


\title{
Remerciements
} de Paris.

Le bélier Mouflon de Corse a été grâcieusement fourni par le Muséum d'Histoire Naturelle

Le texte a été relu par le $D^{r} \mathrm{~S}$. Adalsteinsson (Reykjavik, Islande) et ses observations ont été très constructives.

\section{Summary}

\author{
Hybridization between a corsican Mouflon (Ovis ammon musimon, ScHREBER I872) \\ and various ewe genotypes: Genes for pigmentary color
}

A male Mouflon of Corsica was crossed with ewes of different known color genotypes at $A$ (Agouti) and $E$ (Extension) loci. These loci are the basic elements controlling mammal hair (and sheep fleece) pigmentation.

It was first concluded that the wild male carried a non-muted $E+$ allele in $E$, usually present in domestic sheep except in breeds carrying a black dominant $\left(E^{d} E^{d}\right.$ or $\left.E^{d} E^{+}\right)$. The $E^{+}$allele permits the Agouti genes to be normally expressed.

At the Agouti locus, the wild sheep carried a wild $A^{+}$allele. This allele causes the wild breed pattern, i.e. fawn with black traces and white belly. This allele was recessive in relation to $A^{\text {wh }}$ (red) and dominant with a variable expression in relation to $a$ (black): $5 A+a$ out of 8 had the wild phenotype at birth (as $A^{+} A^{+}$) and 3 had an intermediate phenotype very similar to "black and tan ": black back, white belly. The problem was to determine if the wild allele $A^{+}$ could be confused with an allele already identified in several sheep breeds which gives the "black and $\tan n(A w)$. A definitive answer was not forthcoming, but it seemed that there are two distinct, alleles, even if the expression of one may be imitated by the other $(A+a$ giving the "black and $\tan "$ as $A{ }^{w} a$ or $A{ }^{*} A{ }^{w}$ ), and if the modification of flece structure (going from the wild species to the domestic) may, at least partially, explain the change in gene cxpression.

\section{Références bibliographiques}

Adalsteinsson S., 1960. Inheritance of coat colour in sheep (islandais res. anglais). Freyr, 23-24.

Adalsteinsson S., 1970. Colour inheritance in Icelandic Sheep and relation between colour, fertility and fertilization. J. Agr. res. Icel., 2, 3-135.

Adalsteinsson S., 1974. Colour inheritance in farm animals and its application in selection. Irst World Congr. Genet. appl. Livest. Prod., 1, 29-37.

Berge S., I958. Colour in the Old Norwegian Sheep (norvégien, res. anglais). Meld. Norg. Landbruskshøgsk., 37 (6), I7.

Berge S., 1964. Sheep Colour Genetics (norvégien, rés. anglais). Meld. Norg. Landbrukshøgsk. 43, (6), pp.32.

Berge S., I974. Sheep Colour Genetics. Z. Tierzïcht. Zücht. Biol., 90, 297-32I.

Brooker Mi. G., Dolling C. H. S., I 969 . Pigmentation of sheep. II. The inheritance of colour patterns in black Merinos. Austr. J. Agric. Res., 20, 387-394.

Bunch T. D., Foote W. C., Spillett J. J., 1976. Translocation of acrocentric chromosomes and their implications in the evolution of Sheep (Ovis). Cytogenet. Cell. Genet., 17, I22-I36.

Butarin N. S., 1938. Hybridization between wild mountain Sheep (Ovis Kavelini Sev.) and fine wooled sheep and the problem of creating a domestic breed of mountain sheep (russe, res. anglais). Bull. Acad. Sci. U.R.S.S., Ser. Biol., 853-862.

Hoogschagen P., I967. Hérédité de la couleur noire chez les moutons hollandais (néerlandais). Veeteelt Zuiv. Bericht., 10 (I), 22-27. 
Косн W., I937. Ü̈ber Wildfarbung und ihre Vererbung beim Schaf. Züchtungskunde, 12, 325-328.

Lauvergne J. J., I970. Mise en évidence de l'existence du phénotype noir et feu dans deux nouvclles espèces de Mammifères. Rev. Roum. Biol., (Zool), 15, I I3-1 18.

LAUvergne J. J., I975. Génétique de la couleur de la toison de trois races ovines françaises, Berrichonne, Bizet et Solognote. Ann. Ciénét. Sél. anim., 7, 263-276.

Lauvergne J. J., Adalsteinsson S., i976. Gènes pour la couleur de la toison de la brebis Corse. Ann. Génét. Sél. anim., 8, i $53^{-1} 72$.

NeL J. W., I962. Sheep hybrids from a Nouflon ram and ewes of South African breeds. S. Afr. J. Sci., 58, 299-300.

Nel J. W., 1967. Genetic studies in Karakul Sheep. Ann. Univ. Stellenbosch (Ser. A.), 42, (33), I 53-3I 7 .

Pfeffer P., 1967. Le mouflon de Corse (Ovis ammon musimon Schr., I782); position systématique, écologie et éthologie comparées. Mammalia, 31, Suppl. pp. 25I.

RENDiL J., 1957. Heredity of colour and white marks in farm animals (suédois). Kungl. Skogs. Landbruksakad. Tidskr., 96, 208-263.

Roberts, I93I. Colour inheritance in sheep. VI. The genetic constitution of the Wild Mouflon. J. Genet., 25, I-I6.

Roberts J. A. F., Jenkin T. J., 1926. A premiminary note on reversed badger face pattern in sheep. Welsh J.Agr., 2, 70-73.

SeARle A. G., 1968. Comparative genetics of coat colouv in mammals. Logos Press, Academic Press, London and New-York, 68-69, 75-76, I 89-194.

WARwick B. I., BerRy R. O., Davis S. P., 1957. Inheritance of recessive black in sheep. J. Hered., 48, $255^{-2} 58$.

Young S. P., Mianville R. H., I960. Records of Bighom hybrids. J. Mamm., 41, 523-525. 